\title{
Catechol biosynthesis from glucose in Escherichia coli anthranilate-overproducer strains by heterologous expression of anthranilate 1,2-dioxygenase from Pseudomonas aeruginosa $\mathrm{PAO} 1$
}

Víctor E Balderas-Hernández², Luis G Treviño-Quintanilla , Georgina Hernández-Chávez, Alfredo Martinez', Francisco Bolívar ${ }^{1}$ and Guillermo Gosset ${ }^{1 *}$

\begin{abstract}
Background: The aromatic compound catechol is used as a precursor of chemical products having multiple applications. This compound is currently manufactured by chemical synthesis from petroleum-derived raw materials. The capacity to produce catechol is naturally present in several microbial species. This knowledge has been applied to the generation of recombinant Escherichia coli strains that can produce catechol from simple carbon sources.

Results: Several strains derived from E. coli W3110 trpD9923, a mutant that overproduces anthranilate, were modified by transforming them with an expression plasmid carrying genes encoding anthranilate 1,2-dioxygenase from Pseudomonas aeruginosa PAO1. The additional expression of genes encoding a feedback inhibition resistant version of 3-deoxy-D-arabino-heptulosonate 7-phosphate (DAHP) synthase and transketolase from E. coli, was also evaluated. Generated strains were characterized in complex or minimal medium in shake-flask and fed-batch bioreactor cultures and incubation temperatures ranging from 37 to $28^{\circ} \mathrm{C}$. These experiments enabled the identification of culture conditions for the production of $4.47 \mathrm{~g} / \mathrm{L}$ of catechol with strain W3110 trpD9923, expressing 1,2-dioxygenase, DAHP synthase and transketolase. When considering the amount of glucose consumed, a yield of $16 \%$ was calculated, corresponding to $42 \%$ of the theoretical maximum as determined by elementary node flux analysis.

Conclusions: This work demonstrates the feasibility of applying metabolic engineering for generating E. coli strains for the production of catechol from glucose via anthranilate. These results are a starting point to further optimize environmentally-compatible production capacity for catechol and derived compounds.
\end{abstract}

Keywords: Aromatics, Catechol, Anthranilate, Metabolic engineering, Escherichia coli, Anthranilate 1,2-dioxygenase

\section{Introduction}

Aromatic compounds are used in industry mainly as building blocks for the generation of various valuable products. The synthesis of aromatics using metabolic engineering and synthetic biology is an arising and promising alternative to the conventional chemical production

\footnotetext{
* Correspondence: gosset@ibt.unam.mx

'Departamento de Ingeniería Celular y Biocatálisis, Instituto de Biotecnología, Universidad Nacional Autónoma de México, Apdo, Postal 510-3, Cuernavaca, Morelos CP 62271, Mexico

Full list of author information is available at the end of the article
}

methods. The biotechnological production of aromatic compounds relies mainly on the use of microorganisms that naturally synthesize a broad range of organic compounds, and more importantly, on the utilization of renewable starting materials different from petroleum derivatives. The production of catechol is among one of the multiple examples of how biotechnology is changing traditional chemical synthesis methods. Catechol (1,2dihydroxybenzene) is an aromatic compound used in a variety of applications. It is employed as a reagent for photography, dyestuffs, electroplating, rubber and plastics 
production, also as starting material for the production of insecticides, perfumes and some drugs [1]. The oxidation of phenol and $m$-diisopropylbenzene and the distillation of coal-tar are the main methods for catechol production [1]. However, these procedures are expensive, lengthy and non-environmentally sustainable, due to their characteristics in terms of reactant requirements and reaction conditions. Alternatively, several Pseudomonas species display the capacity of growing utilizing aromatic hydrocarbons, transforming them into catechol or protocatechuate. This metabolic capacity has been employed for producing catechol using microorganisms as biocatalysts [2]. In a Pseudomonas putida strain expressing the enzyme toluene/benzene dioxygenase and lacking catechol 1,2oxygenase and catechol 2,3-oxygenase activities, catechol was produced by benzene oxidation [3]. Also in a Bacillus stearothermophilus, strain BR219, capable of degrading phenol, the addition of tetracycline during the stationary phase resulted in the inactivation of the plasmid encoded catechol 2,3-dioxygenase from the phenol meta pathway of this microorganism, resulting in the accumulation of catechol [4]. Alternative to this strategy, transposon Tn916 was used to disable the catechol 2,3-dioxygenase gene in B. stearothermophilus [5].

The first example of Escherichia coli strains designed for catechol production was based on genetic modifications to cause accumulation of the intermediate 3-dehydroshikimic acid (DHS) and expression of genes encoding heterologous DHS dehydratase and protocatechuic acid decarboxylase. One of these recombinant $E$. coli strains produced catechol with a $33 \%$ yield from glucose [6]. In another example, a $2 \%$ increase in yield of catechol was observed when applying a resin-based extraction process to reduce the toxic effect of this product [7]. In these strains, the genetic modification causing DHS accumulation also results in a multiple auxotrophy for aromatic amino acids and vitamins, therefore, culture medium supplementation is required.

Our group previously generated and characterized a recombinant bacterial system for the production of anthranilate from glucose based on E. coli strain W3110 trpD9923. This strain displays a low anthranilate producing capacity, resulting from a mutation in the $\operatorname{trp} D$ gene (the anthranilate phosphoribosyl transferase function) of the tryptophan operon (Figure 1). Derivatives of this strain were generated by following strategies known to increase carbon flow to the synthesis of the aromatic amino acids. The central metabolism precursors phosphoenolpyruvate (PEP) and erythrose 4-phosphate were redirected into the common aromatic pathway by transforming W3110 trpD9923 with plasmid pJLBaroG ${ }^{\mathrm{fbr}} t k t A$. This plasmid carries the genes encoding a feedback inhibition resistant version of the enzyme 3-deoxy-D-arabino-heptulosonate7-phosphate (DAHP) synthase $\left(\right.$ aro $\left.G^{\mathrm{fbr}}\right)$ and transketolase $(t k t A)$. To avoid consumption of PEP during glucose import, the phosphoenolpyruvate:sugar phosphotransferase system (PTS) was inactivated (Figure 1). One of the generated strains, W3110 trpD9923/pJLBaroG ${ }^{\mathrm{fbr}} t k t A$ produced $14 \mathrm{~g} / \mathrm{L}$ of anthranilate in fed-batch cultivation with glucose [8].

Various microorganisms have enzymes that catalyze mono- and dioxygenation reactions of aromatic substrates such as benzene, phenol, aniline, toluene, anthranilate, among others, to generate catechol, which can be further catabolized via the $\beta$-ketoadipate pathway to generate tricarboxylic acid cycle (TCA) intermediates [9]. The strain P. aeruginosa PAO1 is able to use anthranilate as a carbon and energy source [10]. In this bacterium, via ortho-dihydroxylation followed by spontaneous deamination and decarboxylation, anthranilate is converted to catechol. The hydroxylation reaction is catalyzed by a class IB two-component anthranilate 1,2-dioxygenase (AntDO), which comprises the terminal oxygenase component (AntAB) and the reductase component (AntC) (Figure 1) [11,12].

In this work, we explored the feasibility of producing catechol from glucose via anthranilate with strains having a modification that causes a single auxotrophy for L-Trp. We genetically engineered several anthranilate-producing strains derived from W3110 trpD9923 by expressing the ant $A B C$ genes coding for the AntDO of $P$. aeruginosa PAO1 (Figure 1) and determined their capacity for producing catechol under various culture conditions.

The existence of two alternate approaches for microbial catechol production from simple carbon sources will allow studies to determine which one will result in strains and production processes displaying desirable characteristics such as high strain robustness and specific productivity as well as low culture medium cost. This report will provide initial data allowing such comparisons to be made.

\section{Material and methods}

\section{Strains and plasmids}

The E. coli strains and plasmids used in this study are described in Table 1. E. coli strain W3110 trpD9923 was obtained from the E. coli Genetic Stock Center (Yale University, New Haven, CT). E. coli W3110 trpD9923 strain is a mutant in the $\operatorname{trpD}$ gene of the tryptophan operon obtained by treatment with ultraviolet radiation, thus, it accumulates anthranilate and it is a tryptophan auxotroph [13]. The W3110 trpD9923 PTS $^{-}$strain is a derivative of W3110 trpD9923 with an inactivated PTS operon ( $\left.\Delta p t s H, p t s I, \quad c r r:: \mathrm{Km}^{\mathrm{R}}\right)$ [8]. Plasmid pJLBar$o G^{\mathrm{fbr}} t k t A$ carries the $\operatorname{aro} G^{\mathrm{fbr}}$ and $t k t A$ genes encoding a feedback inhibition resistant version of 3-deoxy-Darabino-heptulosonate 7-phosphate synthase and transketolase, respectively [14]. Plasmid pTrc-ant3 carries the 


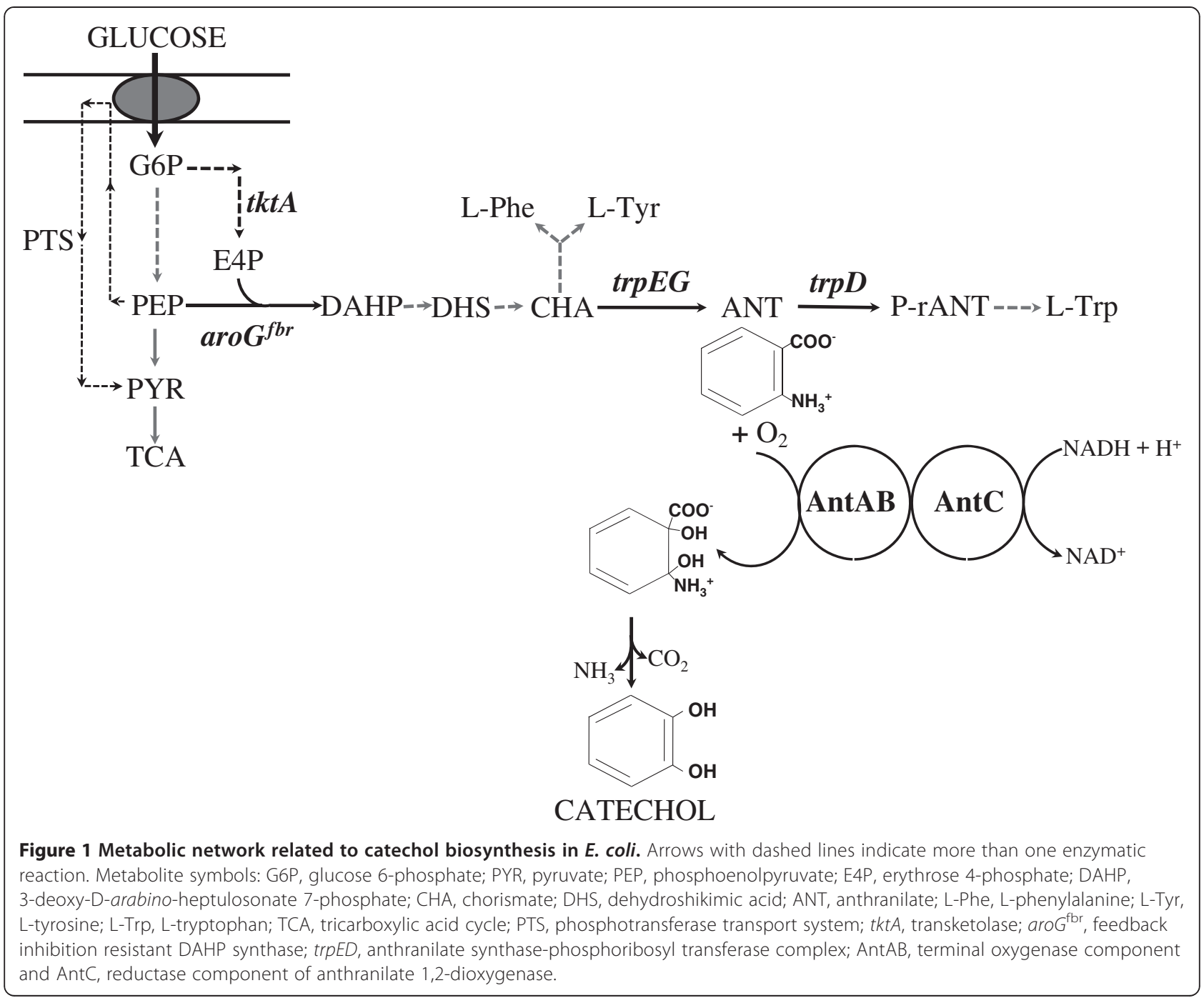

ant $A B C$ genes coding for the anthranilate 1,2-dioxygenase (AntDO) of $P$. aeruginosa PAO1. The genes were amplified by PCR using total DNA from $P$. aeruginosa PAO1 [15] as template and the forward primer 5'-CGAGG CGTTCTAGATCACCGGCGTGAC-3' containing the XbaI site (in bold) and the reverse primer $5^{\prime}$-GTGAGAA
CCCATGGACGCTACCCGCAG-3' containing the NcoI site (in bold). The antABC PCR product (2983 bp) was first cloned into pCR-Blunt II-TOPO plasmid (Invitrogen, Carlsbad, CA) and then subcloned into the pTrc99A plasmid in the sites $\mathrm{XbaI}$ and NcoI, under the control of the trc promoter.

Table 1 Escherichia coli strains and plasmids used in this work

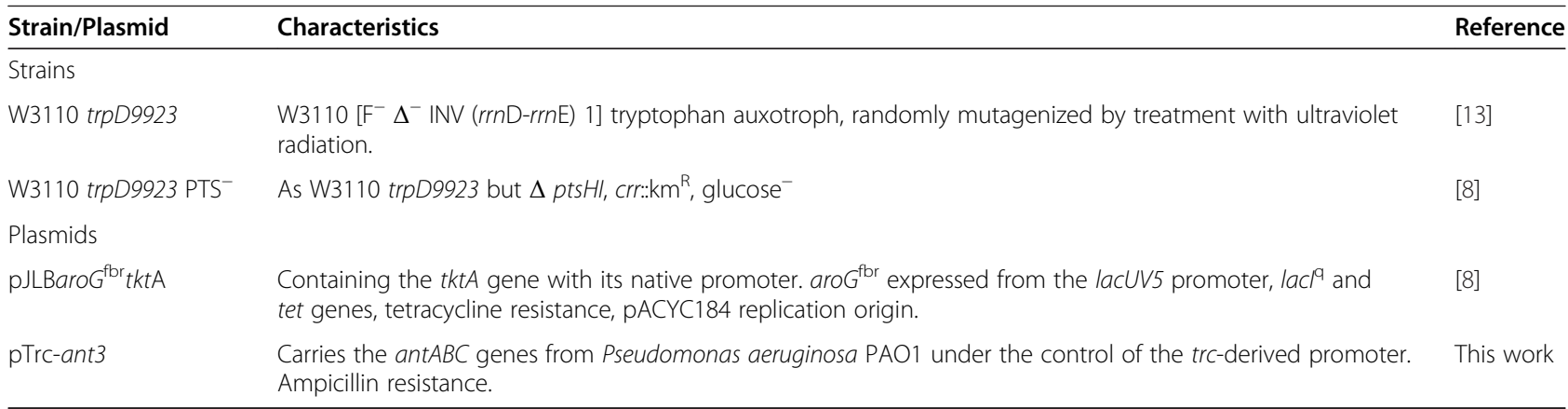




\section{Growth media, inoculum preparation and culture conditions}

Cells were routinely grown in Luria Bertani (LB) broth or LB-agar plates. Experiments in flask cultures were carried out using defined medium, containing $10 \mathrm{~g} / \mathrm{L}$ glucose, 6 g/L Na${ }_{2} \mathrm{HPO}_{4}, 0.5 \mathrm{~g} / \mathrm{L} \mathrm{NaCl}, 3 \mathrm{~g} / \mathrm{L} \mathrm{K \textrm {K } _ { 2 }} \mathrm{PO}_{4}$, $1 \mathrm{~g} / \mathrm{L} \mathrm{NH}_{4} \mathrm{Cl}, 246.5 \mathrm{mg} / \mathrm{L} \mathrm{MgSO}_{4}, 14.7 \mathrm{mg} / \mathrm{L} \mathrm{CaCl}_{2}$ and $10 \mu \mathrm{g} / \mathrm{mL}$ vitamin B1, and supplemented with $20 \mu \mathrm{g} / \mathrm{mL}$ tryptophan. Salts, glucose, vitamin $\mathrm{B} 1, \mathrm{MgSO}_{4}$, tryptophan, and $\mathrm{CaCl}_{2}$ solutions were sterilized separately. Medium for bioreactor cultures contained $3 \mathrm{~g} / \mathrm{L} \mathrm{Na} \mathrm{NaO}_{4}, 3 \mathrm{~g} / \mathrm{L}$ $\mathrm{KH}_{2} \mathrm{PO}_{4}, 1.7 \mathrm{~g} / \mathrm{L}\left(\mathrm{NH}_{4}\right)_{2} \mathrm{HPO}_{4}$ and $1 \mathrm{~mL} / \mathrm{L}$ of trace elements solution. This solution contains $2.7 \mathrm{~g} / \mathrm{L} \mathrm{FeCl}_{3}$, $2 \mathrm{~g} / \mathrm{L} \mathrm{ZnCl} 3,2 \mathrm{~g} / \mathrm{L} \mathrm{CoCl}{ }_{2} \cdot 6 \mathrm{H}_{2} \mathrm{O}, 2 \mathrm{~g} / \mathrm{L} \mathrm{Na}_{2} \mathrm{MoO}_{4} \cdot 2 \mathrm{H}_{2} \mathrm{O}$, $2 \mathrm{~g} / \mathrm{L} \mathrm{CaCl}_{2} \cdot 2 \mathrm{H}_{2} \mathrm{O}, 0.5 \mathrm{~g} / \mathrm{L} \mathrm{H}_{3} \mathrm{BO}_{3}$ and $100 \mathrm{~mL} / \mathrm{L} \mathrm{HCl}$. A fed-batch culture strategy was employed to provide a relatively high amount of glucose $(90 \mathrm{~g} / \mathrm{L})$, divided in feeding stages to avoid osmotic shock effects. Bioreactor culture medium initially contained $30 \mathrm{~g} / \mathrm{L}$ of glucose. A total of two independent pulses of $30 \mathrm{~g} / \mathrm{L}$ glucose, as $25 \mathrm{~mL}$ of $60 \%$ sterile glucose solution, were added to the bioreactor whenever glucose concentration in the medium decreased to $10 \mathrm{~g} / \mathrm{L}$ as measured off-line using an enzymatic analyzer. For some experiments, bioreactor medium was supplemented with $10 \mathrm{~g} / \mathrm{L}$ of yeast extract at the beginning of the experiment and two pulses of $10 \mathrm{~g} / \mathrm{L}$ yeast extract were added. Antibiotics were added to the corresponding cultures at a final concentration of $200 \mu \mathrm{g} / \mathrm{mL}$ ampicillin, $20 \mu \mathrm{g} / \mathrm{mL}$ tetracycline and $30 \mu \mathrm{g} / \mathrm{mL}$ kanamycin during selection, propagation and fermentation stages. Inoculum preparation was started using strain samples from frozen vials that were cultured overnight at $37^{\circ} \mathrm{C}$ in LB-agar plates, a single colony from these plates was used to inoculate baffled shake flasks containing mineral medium supplemented with $0.2 \%$ of glucose and $20 \mu \mathrm{g} / \mathrm{mL}$ tryptophan. After overnight growth at $37^{\circ} \mathrm{C}$ a sample was used for inoculation. For bioreactor cultures experiments, colonies from LB-agar plates were grown in $500 \mathrm{~mL}$ shake flasks with $100 \mathrm{~mL}$ mineral medium supplemented with $0.2 \%$ of glucose, $20 \mu \mathrm{g} / \mathrm{mL}$ tryptophan and $0.1 \%$ of peptone. Flask culture experiments were done in $250 \mathrm{~mL}$ flasks containing $50 \mathrm{~mL}$ of mineral medium, inoculated at an initial optical density at $600 \mathrm{~nm}\left(\mathrm{OD}_{600 \mathrm{~nm}}\right)$ of 0.1 and incubated at $37^{\circ} \mathrm{C}$ and $300 \mathrm{rpm}$ in an orbital shaker (Series 25, New Brunswick Scientific, Inc., NJ). Bioreactor cultures were performed in $1 \mathrm{~L}$ stirred tank bioreactors (Applikon, The Netherlands), using a working volume of $500 \mathrm{~mL}$. Cultures were inoculated at an initial $\mathrm{OD}_{600 \mathrm{~nm}}$ of 0.5 , since a lower inoculum concentration resulted in a very long lag phase. $\mathrm{pH}$ was maintained at 7.0 by automatic addition of a $12.5 \% \mathrm{NH}_{4} \mathrm{OH}$ solution. Airflow was set to $1 \mathrm{vvm}$. Dissolved oxygen tension was measured with a polarographic oxygen electrode (Applisens, Applikon) and maintained above $20 \%$ air saturation during all cultivation period by modifying the impeller speed. The cultivation temperature was controlled at 37, 32, or $28^{\circ} \mathrm{C}$ depending of the experiment as indicated. For cultures of strains carrying plasmid pJLBaroG ${ }^{\mathrm{fbr}} t k t A$ and/or pTrc-ant3, gene induction was started by adding IPTG to a final concentration of $0.1 \mathrm{mM}$ at an $\mathrm{OD}_{600 \mathrm{~nm}}$ of 0.6 for shake flask and 3.0 for bioreactor cultures. Inducer IPTG was employed at a final concentration of $0.1 \mathrm{mM}$ based on experiments showing similar strain performance when compared to conditions having a final concentration of $1 \mathrm{mM}$.

\section{Kinetic parameters calculation}

For the characterization of the strains used in this work, specific rates of growth $(\mu)$, glucose consumption $\left(q_{\mathrm{Glc}}\right)$, anthranilate production $\left(q_{\text {Ant }}\right)$ and catechol production $\left(q_{\text {Cat }}\right)$, as well as yields of anthranilate $\left(\mathrm{Y}_{\mathrm{Ant} / \mathrm{Glc}}\right)$ and catechol $\left(\mathrm{Y}_{\mathrm{Cat} / \mathrm{Glc}}\right)$ on glucose were determined. $\mu$ and $q_{\mathrm{Glc}}$ were calculated during the exponential growth phase. Since growth rates and production kinetics of catechol and anthranilate differed among studied strains, $q_{\text {Ant }}$, $q_{\text {Cat }}, \mathrm{Y}_{\text {Ant/Glc }}$, and $\mathrm{Y}_{\text {Cat/Glc }}$ were calculated considering only the catechol or anthranilate production phase, defined as the time period starting when catechol or anthranilate was detected up to the point when a sharp decrease in product accumulation was observed. Flask cultures were performed in triplicate and bioreactor cultures in duplicate. The values reported represent the mean of all performed experiments and standard errors are shown in tables.

\section{Analytical methods}

Biomass concentration was measured as $\mathrm{OD}_{600 \mathrm{~nm}}$ using a spectrophotometer (Beckman DU-70, Palo Alto, CA) and converted to dry cell weight (DCW) considering that $1 \mathrm{OD}_{600 \mathrm{~nm}}=0.37 \mathrm{~g}_{\mathrm{DCW}} / \mathrm{L}$ [16]. Samples taken during cultivation period were centrifuged at $10000 \mathrm{rpm}$ for $2 \mathrm{~min}$. Supernatant was filtered using $0.45 \mu \mathrm{m}$ syringefilter and stored at $-20^{\circ} \mathrm{C}$ for subsequent analysis. Glucose was determined using an enzymatic analyzer (YSI 2700, YSI Life Sciences, OH). Acetate was determined by high performance liquid chromatography (HPLC) (Waters, Milford, MA), using an Aminex HPX-87H column (300x7.8 mm; Bio-Rad, Hercules, CA); running conditions were $5 \mathrm{mM} \mathrm{H}_{2} \mathrm{SO}_{4}$ as mobile phase, flow of $0.5 \mathrm{~mL} / \mathrm{min}$ and temperature of $50^{\circ} \mathrm{C}$. Detection was performed by photodiode array at $210 \mathrm{~nm}$. Catechol and anthranilate were determined from the culture medium and not from biomass, since the former is the fraction available for recovery. The method employed was HPLC (Agilent Technologies, Palo Alto, CA) using a Synergy Hydro $\mathrm{C}_{18} 4 \mu \mathrm{m}$ column (4.6x150 mm, Phenomenex, Torrance, CA); running conditions were $0.1 \%$ trifluoroacetic acid in $40 \%$ methanol as mobile phase, flow of $0.5 \mathrm{~mL} / \mathrm{min}$. Detection 
was performed by photodiode array at $330 \mathrm{~nm}$ for anthranilate and at $280 \mathrm{~nm}$ for catechol. The maximum theoretical yield for synthesis of catechol or anthranilate from glucose corresponds to $0.376 \mathrm{~g} / \mathrm{g}$ and was determined by applying elementary mode flux analysis using METATOOL [17].

\section{Results}

Production of catechol in shake-flask cultures with derivatives of $E$. coli W3110 trpD9923

The enzyme AntDO from $P$. aeruginosa PAO1, encoded by ant $A B C$ genes, catalyzes the conversion of anthranilate to catechol. To generate an $E$. coli strain for the production of catechol via anthranilate, the ant $A B C$ genes were amplified from $P$. aeruginosa PAO1 and cloned in an expression vector, thus generating plasmid pTrc-ant3. Based on data described by Balderas et al. (2009), strains W3110 trpD9923, W3110 trpD9923 PTS ${ }^{-}$and W3110 trpD9923/pJLBaroG ${ }^{\mathrm{fbr}}$ tktA were selected for this study since they displayed a wide range of anthranilate production capacity from glucose. These three strains were transformed with plasmid pTrc-ant3.

To determine if expression of AntDO in these strains would cause the transformation of anthranilate to catechol, they were characterized in shake flask cultures using mineral medium supplemented with $10 \mathrm{~g} / \mathrm{L}$ glucose. (Figure 2 and Table 2). The strains W3110 trpD9923/pTrc-ant3 and W3110 trpD9923/pJLBaroG ${ }^{\mathrm{fbr}}$ tktA/pTrc-ant3 showed similar $\mu$ values, whereas $q_{\mathrm{Glc}}$ was lower for the latter strain. As a result of PTS inactivation, strain W3110 trpD9923 $\mathrm{PTS}^{-} / \mathrm{pTrc}$-ant3 showed the lowest $\mu$ and $q_{\mathrm{Glc}}$. Catechol was detected in cultures with all strains, where the $Y_{\text {Cat/Glc }}$ values corresponded to 24,27 and $13 \%$ of the theoretical maximum for strains W3110 trpD9923 $\mathrm{PTS}^{-} / \mathrm{p}$ Trc-ant3, W3110 trpD9923/pJLBaroG ${ }^{\mathrm{fbr}} t k t A /$ pTrc-ant3 and W3110 trpD9923/pTrc-ant3, respectively. Anthranilate was also detected in cultures with strains W3110 trpD9923/pJLBaro ${ }^{\mathrm{fbr}}{ }^{\text {tktA/pTrc-ant3 and }}$ W3110 trpD9923/pTrc-ant3, indicating its incomplete conversion to catechol. Considering that W3110 trpD9923/ pJLBaroG ${ }^{\mathrm{fbr}} t k t A / \mathrm{p}$ Trc-ant3 showed the highest catechol production capacity, it was selected for further characterization in bioreactor cultures.

\section{Effect of media composition on catechol production in fed-batch bioreactor cultures}

Strain W3110 trpD9923/pJLBaroG ${ }^{\mathrm{fbr}} t k t A / \mathrm{pTrc}-a n t 3$ was evaluated in fed batch cultures using M9 medium supplemented with $90 \mathrm{~g} / \mathrm{L}$ of total fed glucose and yeast extract $30 \mathrm{~g} / \mathrm{L}$ at $37^{\circ} \mathrm{C}$. These culture conditions were chosen since they enabled the production of $14 \mathrm{~g} / \mathrm{L}$ of anthranilate by strain W3110 trpD9923/pJLBaroG ${ }^{\mathrm{bbr}}$ tktA [8]. Strain W3110 trpD9923/pJLBaroG ${ }^{\mathrm{fbr}} t k t A / \mathrm{pTrc}-a n t 3$ displayed a $\mu$ of $0.17 \pm 0.03 \mathrm{~h}^{-1}$ and a maximum biomass concentration of $10.44 \pm 1.14 \mathrm{~g} / \mathrm{L}$ at $30 \mathrm{~h}$ of culture (Figure 3, Table 3). Under these conditions, this strain produced $2.51 \pm 0.21 \mathrm{~g} / \mathrm{L}$ of catechol and $2.86 \pm 0.09 \mathrm{~g} / \mathrm{L}$ of anthranilate.

Aromatic amino acids present in yeast extract are known to participate in transcriptional repression of genes from the common aromatic and tryptophan biosynthetic pathways, as well as having an inhibitory effect on enzymes from these pathways [18]. To determine if yeast extract has a negative impact on anthranilate and catechol synthesis capacity, further characterization of W3110 trpD9923/ pJLBaro ${ }^{\mathrm{fbr}} t k t A / \mathrm{p}$ Trc-ant3 was performed using mineral M9 medium supplemented with $90 \mathrm{~g} / \mathrm{L}$ of total fed glucose but lacking yeast extract. To determine anthranilate production under these culture conditions, strain W3110 trpD9923/pJLBaroG ${ }^{\mathrm{fbr}} t k t A$ was also included in these experiments. Strains W3110 trpD9923/pJLBaroG ${ }^{\mathrm{fbr}}{ }^{\mathrm{t} t} \mathrm{t} A$ and W3110 trpD9923/pJLBaroG ${ }^{\mathrm{fbr}}$ tktA/pTrc-ant3 displayed lower final biomass concentrations when compared with cultures supplemented with yeast extract. Under these growth conditions the final concentration of anthranilate produced by strain W3110 trpD9923/ pJLBaroG ${ }^{\mathrm{fbr}} t k t A$ was $8.18 \pm 0.08 \mathrm{~g} / \mathrm{L}$ with a $Y_{\mathrm{Ant} / \mathrm{Glc}}$ of $0.17 \pm 0.08 \mathrm{~g}_{\text {Ant }} / \mathrm{g}_{\mathrm{Glc}}$ (Table 3). Strain W3110 trpD9923/ pJLBaro $G^{\mathrm{fbr}} t k t A / \mathrm{p}$ Trc-ant3 accumulated $2.81 \pm 0.15 \mathrm{~g} / \mathrm{L}$ of catechol and $2.12 \pm 0.08 \mathrm{~g} / \mathrm{L}$ of anthranilate, with a $Y_{\text {Cat/Glc }}$ corresponding to $21 \%$ of the theoretical maximum.

\section{Effect of incubation temperature on catechol production}

The incubation temperature is a parameter that can have an important effect on the production level and activity of heterologous proteins expressed in E. coli, as well as the general cell physiology [19]. Therefore, to determine the effect of incubation temperature on catechol production, bioreactor cultures with strains W3110 trpD9923/ pJLBaro $G^{\mathrm{fbr}}$ tktA and W3110 trpD9923/pJLBaroG ${ }^{\mathrm{fbr}} t k t A /$ pTrc-ant3 using mineral medium supplemented with a total of $90 \mathrm{~g} / \mathrm{L}$ of glucose were carried out at 37, 32 and $28^{\circ} \mathrm{C}$.

Final biomass accumulation observed for strain W3110 trpD9923/pJLBaroG ${ }^{\mathrm{fbr}}$ tktA was unaffected at the different evaluated temperatures (Table 3). Anthranilate production and $Y_{\text {Ant/Glc }}$ from cultures done at $32^{\circ} \mathrm{C}$ showed a slight increment when compared to experiments at $37^{\circ} \mathrm{C}$, whereas at $28^{\circ} \mathrm{C}$ these parameters displayed a lower value (Table 3 ).

Growth and catechol production profiles for strain W3110 trpD9923/pJLBaroG ${ }^{\mathrm{fbr}}$ tktA/pTrc-ant3 were differentially affected by the incubation temperatures employed. A reduction in $q_{\mathrm{Glc}}$ was evident at 32 and $28^{\circ} \mathrm{C}$. The final catechol concentration in cultures at $28^{\circ} \mathrm{C}$ was the lowest observed. At $32^{\circ} \mathrm{C}, 4.47 \pm 0.16 \mathrm{~g} / \mathrm{L}$ of catechol and $2.89 \pm$ $0.15 \mathrm{~g} / \mathrm{L}$ of anthranilate were accumulated. The $Y_{\text {Cat } / \mathrm{Glc}}$ was $0.16 \pm 0.02 \mathrm{~g}_{\mathrm{Cat}} / \mathrm{g}_{\mathrm{Glc}}$, 2-fold higher than that observed 


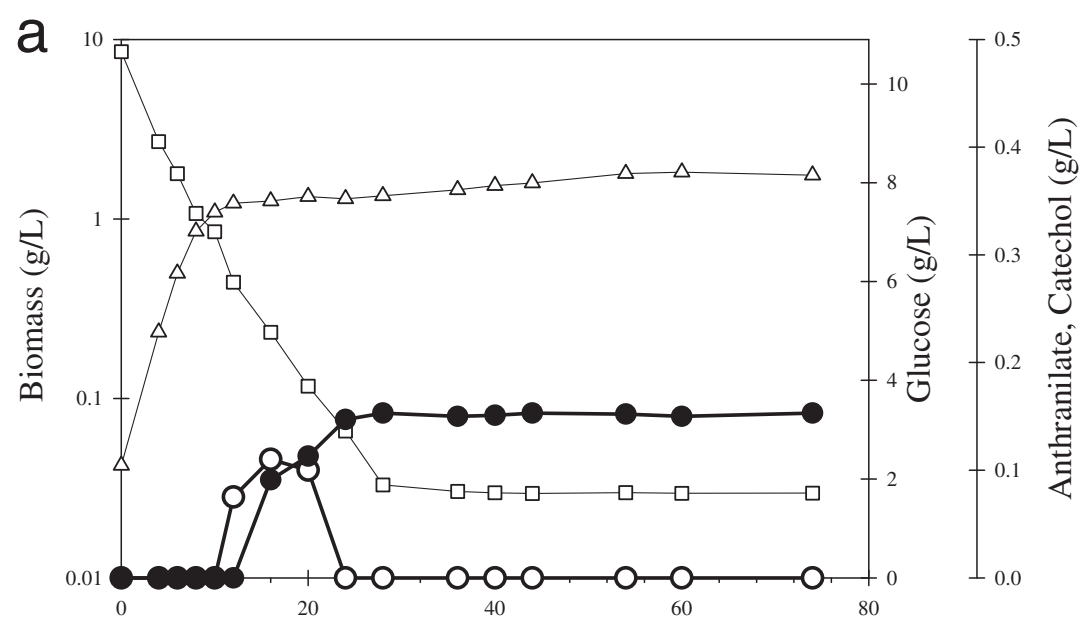

Time (h)

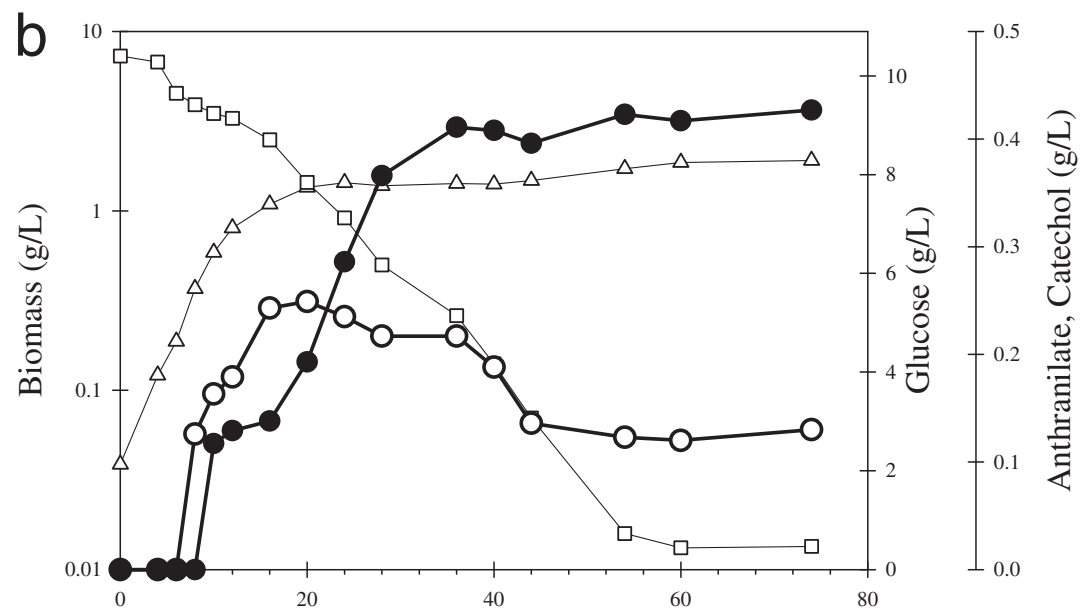

Time (h)

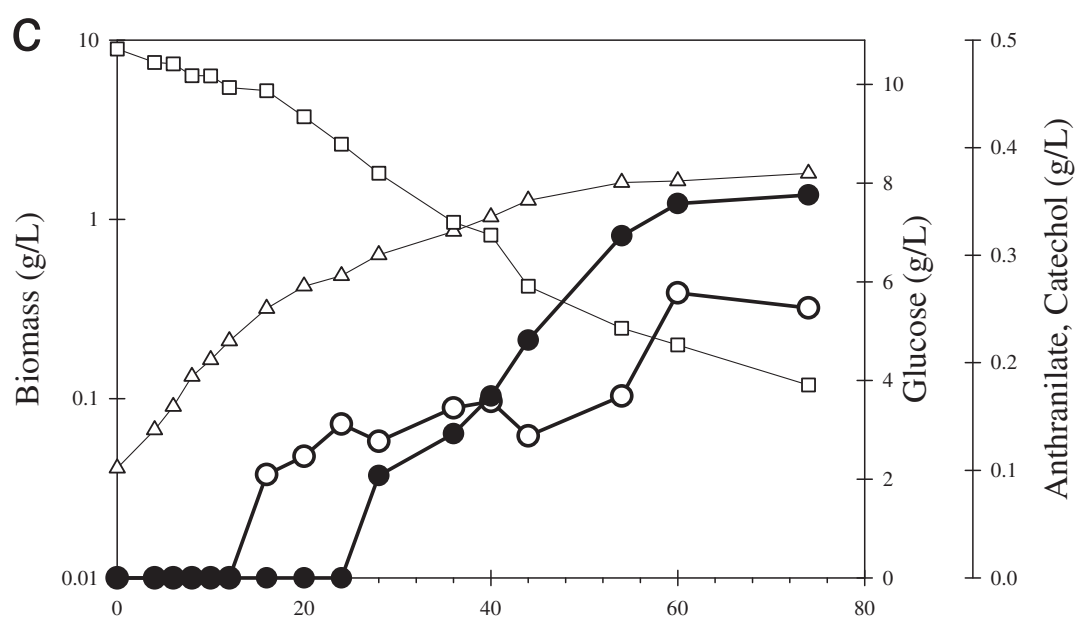

Time (h)

Figure 2 Flask cultures of E. coli W3110 trpD9923 derivative strains. a) 9923/pTrc-ant3, b) 9923/pJLBaroG $G^{\mathrm{fbr}}$ tktA/pTrc-ant3 and c) 9923 PTS /pTrc-ant3. Open triangles; biomass (g/L), open squares; glucose (g/L), open circles; anthranilate (g/L), closed circles; catechol (g/L). 
Table 2 Production of catechol in flask cultures by $E$. coli 9923 derivatives

\begin{tabular}{|c|c|c|c|c|c|c|c|c|}
\hline Strain & $\begin{array}{l}\text { Biomass } \\
\left.\text { (g } \mathrm{g}_{\mathrm{DCW}} / \mathrm{L}\right)\end{array}$ & $\mu\left(h^{-1}\right)$ & $q_{\mathrm{Glc}}\left(\mathrm{g}_{\mathrm{Glc}} / \mathrm{g}_{\mathrm{DCW}} \cdot \mathrm{h}\right)$ & $q_{\text {Ant }}\left(g_{\text {Ant }} / g_{D C w} \cdot h\right)$ & $\begin{array}{l}\text { Anthranilate } \\
(\mathrm{g} / \mathrm{L})\end{array}$ & $\begin{array}{l}\text { Catechol } \\
(\mathrm{g} / \mathrm{L})\end{array}$ & $q_{\mathrm{Cat}}\left(\mathrm{g}_{\mathrm{Cat}} / \mathrm{g}_{\mathrm{DCW}} \cdot \mathrm{h}\right)$ & $\begin{array}{l}\mathrm{Y}_{\mathrm{Cat} / \mathrm{Glc}} \\
\left(\mathbf{g}_{\mathrm{Cat}} / \mathbf{g}_{\mathrm{Glc}}\right)\end{array}$ \\
\hline W3110 9923/ pTrc-ant3 & $1.69 \pm 0.06$ & $0.21 \pm 0.01$ & $0.83 \pm 0.02$ & $0.05 \pm 0.01$ & $0.00 \pm 0.00$ & $0.15 \pm 0.00$ & $0.05 \pm 0.01$ & $0.05 \pm 0.00$ \\
\hline $\begin{array}{l}\text { W3110 } 9923 \\
\text { PTS }^{-} / \text {pTrc-ant3 }\end{array}$ & $1.58 \pm 0.03$ & $0.08 \pm 0.00$ & $0.21 \pm 0.05$ & $0.03 \pm 0.01$ & $0.22 \pm 0.01$ & $0.37 \pm 0.05$ & $0.03 \pm 0.01$ & $0.09 \pm 0.01$ \\
\hline $\begin{array}{l}\text { W3110 9923/ pJLBaroG }{ }^{\mathrm{fbr}} \\
\text { tktA/pTrc-ant3 }\end{array}$ & $1.83 \pm 0.01$ & $0.18 \pm 0.02$ & $0.39 \pm 0.07$ & $0.07 \pm 0.02$ & $0.15 \pm 0.03$ & $0.41 \pm 0.03$ & $0.07 \pm 0.02$ & $0.10 \pm 0.01$ \\
\hline
\end{tabular}

Cultures were carried out in $\mathrm{M} 9$ mineral medium at $37^{\circ} \mathrm{C}$.

in cultures incubated at $37^{\circ} \mathrm{C}$ and corresponding to $42 \%$ of the theoretical maximum (Figure 3, Table 3).

\section{Discussion}

Several microbial species can produce catechol by transformation of various aromatic substrates [2,3]. However, a drawback of such approach is that petroleum constitutes the raw material for synthesizing the aromatic substrates employed in these production processes. For this reason, there is considerable interest in the search of alternatives for the sustainable total synthesis of catechol employing renewable carbon sources as raw material. Production of catechol from a renewable starting material has been achieved by engineering metabolism in $E$. coli. The transformation of intermediate DHS to catechol via protocatechuic acid results from blocking the common aromatic pathway and expressing heterologous DHS dehydratase and protocatechuic acid decarboxylase activities [20]. Further strain improvement by increasing carbon flow to the common aromatic pathway has led to the production of catechol with a high yield from glucose [6].

In the present study, an alternate route for catechol production was developed by the heterologous expression of genes encoding AntDO from $P$. aeruginosa in derivatives of $E$. coli W3110 trpD9923, a mutant that overproduces anthranilate. When transformed with plasmid pTrc-ant3, strain W3110 trpD9923 produced $0.15 \mathrm{~g} / \mathrm{L}$ of catechol from glucose, thus showing AntDO catalytic activity in $E$. coli. When genes aro $G^{\mathrm{fbr}}$ and $t k t A$ were overexpressed in addition to ant $A B C$, a 2.7-fold increase in the final catechol concentration was observed, as well as the accumulation of $0.15 \mathrm{~g} / \mathrm{L}$ anthranilate. This result is explained considering the expected increase in carbon flow to the common aromatic pathway caused by higher level activities of DAHP synthase and transketolase as it has been previously reported [14,21]. The observed accumulation of anthranilate under these conditions indicates that AntDO activity is not sufficient for consuming this precursor at the rate it is being

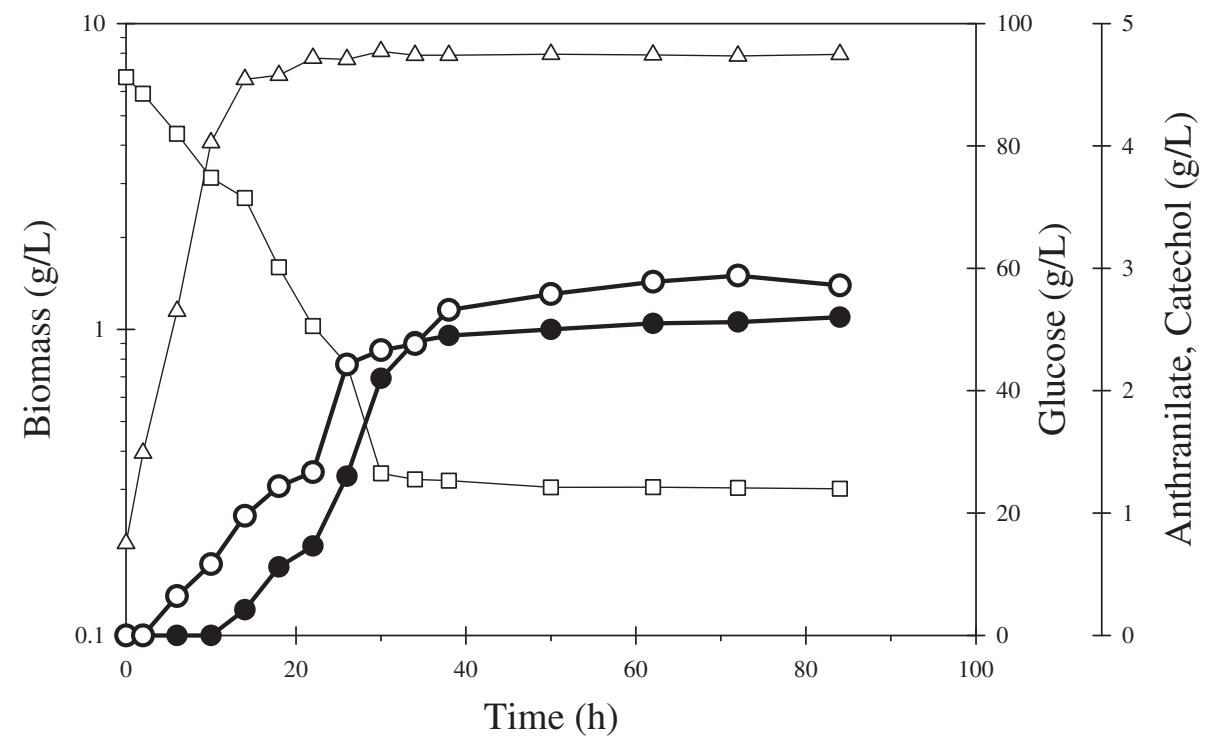

Figure 3 Batch culture of strain W3110trpD9923/pJLBaroG ${ }^{\text {fbr }}$ tktA/pTrc-ant3. Mineral medium supplemented with $90 \mathrm{~g} / \mathrm{L}$ of glucose and $30 \mathrm{~g} / \mathrm{L}$ of yeast extract. Incubation temperature: $37^{\circ} \mathrm{C}$. Open triangles; biomass ( $\left.\mathrm{g} / \mathrm{L}\right)$, open squares; glucose $(\mathrm{g} / \mathrm{L})$, open circles; anthranilate $(\mathrm{g} / \mathrm{L})$, closed circles; catechol ( $\mathrm{g} / \mathrm{L})$. 
Table 3 Production of catechol in bioreactor cultures by $E$. coli 9923 derivatives

\begin{tabular}{|c|c|c|c|c|c|c|c|c|c|}
\hline Culture conditions/Strain & $\begin{array}{l}\text { Biomass } \\
\left(\mathrm{g}_{\mathrm{DCw}} / \mathrm{L}\right) \\
\end{array}$ & $\mu\left(h^{-1}\right)$ & $q_{\mathrm{GIC}}\left(\mathrm{g}_{\mathrm{GI}} / \mathrm{g}_{\mathrm{DCW}} \cdot \mathrm{h}\right)$ & $q_{\text {Ant }}\left(g_{\text {Ant }} / g_{\mathrm{DCW}} \cdot h\right)$ & $q_{\mathrm{Cat}}\left(\mathrm{g}_{\mathrm{Cat}} / \mathrm{g}_{\mathrm{DCW}} \cdot \mathrm{h}\right)$ & Anthranilate (g/L) & Catechol (g/L) & $\begin{array}{l}Y_{\text {Ant } / G l c} \\
\left(g_{\text {Ant }} / g_{\text {Glc }}\right)\end{array}$ & $\begin{array}{l}\mathrm{Y}_{\text {Cat/Glc }} \\
\left(\mathrm{g}_{\mathrm{Cat}} / \mathbf{g}_{\mathrm{Glc}}\right)\end{array}$ \\
\hline \multicolumn{10}{|l|}{$37^{\circ} \mathrm{C}, \mathrm{M} 9+$} \\
\hline \multicolumn{10}{|l|}{$30 \mathrm{~g} / \mathrm{L}$ Yeast Extract } \\
\hline W31 109923/pJLBaroG Gbr tktA* & $18.54 \pm 0.19$ & $0.18 \pm 0.01$ & $0.14 \pm 0.05$ & $0.02 \pm 0.01$ & - & $14.00 \pm 0.07$ & - & $0.20 \pm 0.00$ & - \\
\hline W31 10 9923/pJLBaroG $G^{\mathrm{fbr}}$ tktA/pTrc-ant3 & $10.44 \pm 1.14$ & $0.17 \pm 0.03$ & $0.13 \pm 0.02$ & $0.02 \pm 0.00$ & $0.02 \pm 0.01$ & $2.86 \pm 0.09$ & $2.51 \pm 0.21$ & $0.09 \pm 0.02$ & $0.07 \pm 0.02$ \\
\hline \multicolumn{10}{|l|}{$37^{\circ} \mathrm{C}, \mathrm{M} 9$} \\
\hline W31 10 9923/pJLBaroG Gbr tktA & $7.66 \pm 0.64$ & $0.17 \pm 0.01$ & $0.12 \pm 0.03$ & $0.02 \pm 0.00$ & - & $8.18 \pm 0.08$ & - & $0.17 \pm 0.01$ & - \\
\hline W31 10 9923/pJLBaroG $G^{\text {fbr }}$ tktA/pTrc-ant3 & $7.13 \pm 0.37$ & $0.16 \pm 0.02$ & $0.11 \pm 0.01$ & $0.02 \pm 0.00$ & $0.02 \pm 0.00$ & $2.12 \pm 0.08$ & $2.81 \pm 0.15$ & $0.09 \pm 0.01$ & $0.08 \pm 0.02$ \\
\hline \multicolumn{10}{|l|}{$32^{\circ} \mathrm{C}, \mathrm{M} 9$} \\
\hline W31 10 9923/pJLBaroG GbrtktA & $7.51 \pm 0.44$ & $0.14 \pm 0.01$ & $0.09 \pm 0.01$ & $0.02 \pm 0.00$ & - & $8.61 \pm 0.06$ & - & $0.18 \pm 0.02$ & - \\
\hline W31 10 9923/pJLBaroG $G^{\text {fbr }}$ tktA/pTrc-ant3 & $6.92 \pm 0.27$ & $0.13 \pm 0.01$ & $0.07 \pm 0.02$ & $0.02 \pm 0.00$ & $0.02 \pm 0.01$ & $2.89 \pm 0.15$ & $4.47 \pm 0.16$ & $0.10 \pm 0.02$ & $0.16 \pm 0.02$ \\
\hline \multicolumn{10}{|l|}{$28^{\circ} \mathrm{C}, \mathrm{M} 9$} \\
\hline W31 10 9923/pJLBaroG $G^{\mathrm{fbr}}$ tktA & $7.37 \pm 0.77$ & $0.10 \pm 0.01$ & $0.08 \pm 0.01$ & $0.01 \pm 0.00$ & - & $6.28 \pm 0.10$ & - & $0.11 \pm 0.01$ & - \\
\hline W31 10 9923/pJLBaroG Gbr tktA/pTrc-ant3 & $6.75 \pm 0.34$ & $0.12 \pm 0.01$ & $0.06 \pm 0.00$ & $0.01 \pm 0.00$ & $0.01 \pm 0.01$ & $2.21 \pm 0.14$ & $1.83 \pm 0.13$ & $0.09 \pm 0.02$ & $0.06 \pm 0.01$ \\
\hline
\end{tabular}


produced. This is not observed in cultures with strain W3110 trpD9923/pTrc-ant3 since the rate of anthranilate production is lower, therefore, this substrate can be completely transformed to catechol by AntDO.

Inactivation of PTS in strain W3110 trpD9923 has been reported to cause an increase in the anthranilate yield from glucose [8]. Strain W3110 trpD9923 $\mathrm{PTS}^{-} / \mathrm{p}$ Trcant3, displayed a 2.5-fold increase in catechol titer when compared to $\mathrm{PTS}^{+}$strain W3110 trpD9923/pTrc-ant3. This result can be explained considering that aromatics precursor PEP is not consumed during glucose import in this mutant $[14,22]$. Therefore, a higher carbon flux to the common aromatic pathway is expected. Further improvement in the production performance for this strain could result from overexpression of genes $\operatorname{aroG}^{\mathrm{fbr}}$ and $t k t A$. However, experiments with strain W3110 trpD9923 PTS $^{-}$ revealed a negative effect on anthranilate production parameters when this strain was transformed with plasmid pJLBaroG ${ }^{\mathrm{fbr}} t k t A$ [8]. This result can be explained considering that the $\mathrm{PTS}^{-}$strain cannot cope with the metabolic burden resulting from plasmid replication and the high level expression of genes $\operatorname{aroG}^{\mathrm{fbr}}$ and $t k t A$, since its capacity to consume glucose is lower, when compared to a $\mathrm{PTS}^{+}$strain. It remains to be determined if fine-tuning of expression level of these genes could result in lower metabolic burden and increased production capacity in the PTS $^{-}$strain.

Lower catechol and anthranilate production levels were observed when comparing rich to minimal medium cultures. This result is consistent with allosteric inhibition by tryptophan exhibited by the enzyme anthranilate synthase [23]. Strain W3110 trpD9923 is a tryptophan auxotroph, therefore, this amino acid must be supplemented when employing a minimal medium. In the cultures performed in this study, the concentration of supplemented tryptophan was $20 \mu \mathrm{g} / \mathrm{mL}$. The results observed in these experiments indicate that such tryptophan concentration is sufficient for allowing growth of the trpD mutant but not high enough to inhibit activity of anthranilate synthase.

Culture temperature has been reported to have an important effect on the yield and activity of heterologous proteins expressed in E. coli. Low growth temperatures can result in improved protein folding and reduced protease expression in the host strain [24,25]. AntDO activity was indirectly detected as a result of catechol synthesis, however, the AntDO proteins expressed in the strains in this study could not be identified in protein electrophoresis experiments, likely because expression level is below the limit of visual detection (results not shown). Therefore, it is not possible to determine if growth at temperatures lower than $37^{\circ} \mathrm{C}$ had an impact on the level of AntDO. The observed results suggest a higher AntDO activity at $32^{\circ} \mathrm{C}$ when compared to other culture temperatures. However, different growth temperatures could also alter cellular carbon flux distribution, having a positive or negative impact on catechol production. Therefore, to ascertain the mechanism(s) involved in the observed effects of growth temperature on catechol production capacity, further physiological strain characterization and in vitro analysis of AntDO protein level and activity should be performed.

The mechanisms most frequently cited to explain the toxicity of catechol in $E$. coli cells are: $(i)$ the generation of reactive oxygen species (ROS) by redox reactions; (ii) DNA oxidative damage; (iii) protein damage by sulphydryl arylation or oxidation; and (iv) interference with electron transport in energy transducing membranes [26]. A negative effect of catechol on E. coli growth and production capacity for the aromatic intermediate DHS has been demonstrated starting at $0.275 \mathrm{~g} / \mathrm{L}$ and $2.75 \mathrm{~g} / \mathrm{L}$, respectively [7]. In the bioreactor cultures performed in this study, catechol reached final concentrations spanning a range of 1.83 to $4.47 \mathrm{~g} / \mathrm{L}$. Therefore, a toxic effect of catechol is expected under these conditions, this explains the lower observed $\mu$ when comparing cultures of anthranilate and catechol producer strains. This might also explain why the amount of catechol + anthranilate was lower when comparing cultures with strain W3110 trpD9923/ pJLBaro $G^{\mathrm{fbr}} t k t A$ where only anthranilate was produced, with cultures of strains expressing AntDO. A solution to this issue could be the use of in situ catechol extraction methods by employing an anion-exchange resin, as it has been reported [7].

Important differences exist concerning strain characteristics and catechol production efficiency when comparing synthesis routes starting from either DHS or anthranilate. When considering the catechol synthesis route starting from DHS, the maximum theoretical yield from glucose is $0.686 \mathrm{~g}_{\mathrm{Cat}} / \mathrm{g}_{\mathrm{Glc}}$, Five enzyme catalyzed steps are required to transform DHS to anthranilate. In one of these steps, PEP is consumed for the synthesis of intermediate 5-enolpyruvylshikimate-3-phosphate. Considering this extra carbon atoms requirement, the maximum theoretical yield from glucose for catechol synthesis from anthranilate corresponds to $0.376 \mathrm{~g}_{\mathrm{Cat}} / \mathrm{g}_{\mathrm{Glc}}$. On the other hand, the inactivation of gene aroE encoding SHIK dehydrogenase to cause DHS accumulation results in a deficiency to synthesize the aromatic amino acids and vitamins [6]. This multiple auxotrophy is a potential problem when employing a minimal medium in a production process. However, this deficiency can be circumvented by supplementing the culture medium with the required aromatic compounds or the intermediate shikimate. In contrast, inactivation of $\operatorname{trp} D$ causes a tryptophan auxotrophy, therefore, this amino acid must be supplemented when culturing in minimal medium. Considering these important differences, it remains to be determined which of the two catechol-production routes, based on DHS or 
anthranilate as starting precursors, is more costeffective under industrial production conditions.

A yield of catechol from glucose of $33 \%$ has been reported with strains having the production route starting from DHS [6]. In another report, the yield value was increased from 5 to $7 \%$ when resin-based extraction of catechol was performed [7]. In this work, the highest observed catechol yield from glucose was $16 \%$ with strain W3110 trpD9923/pJLBaroG ${ }^{\mathrm{fbr}}$ tktA/pTrc-ant3 (Table 3). However, this strain also accumulated anthranilate with a $10 \%$ yield from glucose. Therefore, it would be possible to further improve strain performance by increasing AntDO activity to promote the complete transformation of anthranilate to catechol and to improve the production process if a catechol extraction method is applied to reduce toxicity effects.

A novel approach for synthesis of the chemical precursor muconic acid from simple carbon sources in E. coli has been recently described [27]. This method is based on the transformation of anthranilate to catechol and then to muconic acid as a result of expression of heterologous AntDO and catechol 1,2-dioxygenase activities. Pathway optimization led to a strain that accumulated $689 \mathrm{mg} / \mathrm{L}$ of anthranilate and further modification resulted in a strain that produced $389 \mathrm{mg} / \mathrm{L}$ of muconic acid under shake-flask culture conditions. Although catechol was produced from anthranilate as a precursor under the studied conditions, no catechol accumulation in the culture medium was detected since it was completely transformed to muconic acid [27]. Considering the common elements in the metabolic engineering strategies for catechol and muconic acid production, it might be possible to adapt some of the strains and culture conditions developed here, to improve a process for muconic acid biosynthesis.

The strains and culture conditions developed in this work constitute a starting point for the generation of production processes for the synthesis of catechol from glucose at grams scale. This knowledge can be useful for devising and improving production processes for useful compounds derived from catechol.

\section{Competing interests}

The authors declare that they have no competing interests.

\section{Authors' contributions}

VEBH participated in recombinant strain construction and carried out the shake flask and bioreactor production experiments. LGTQ carried out gene cloning and heterologous expression experiments. GHC performed the quantification of substrate and microbial culture products. VEBH, AM and FB participated in analysis of the data. GG coordinated the study. GG and VEBH wrote the manuscript. All authors read and approved the final manuscript.

\section{Acknowledgements}

This work was supported by CONACyT grant 177568. We thank Mercedes Enzaldo for technical assistance.

\section{Author details}

'Departamento de Ingeniería Celular y Biocatálisis, Instituto de Biotecnología, Universidad Nacional Autónoma de México, Apdo, Postal 510-3, Cuernavaca, Morelos CP 62271, Mexico. ${ }^{2}$ Laboratorio de Biología Integrativa de Plantas y Microorganismos, Unidad Académica de Ciencias Biológicas, Universidad Autónoma de Zacatecas, Av. Preparatoria s/n, Col., Agronómica CP 98066Zacatecas, Mexico. ${ }^{3}$ Departamento de Tecnología Ambiental, Universidad Politécnica del Estado de Morelos, Jiutepec, Morelos, Mexico.

Received: 5 June 2014 Accepted: 4 September 2014

Published online: 04 October 2014

\section{References}

1. Fiegel H, Voges HW, Hamamoto T, Umemura S, I wata T, Miki H, Fujita Y, Buysch HJ, Garbe D, Paulus W: Phenol Derivatives. In Wiley NCH, Weinheim: Ullmann's Encyclopedia of Industrial Chemistry; 2000.

2. Tao Y, Fishman A, Bentley WE, Wood TK: Oxidation of benzene to phenol, catechol, and 1,2,3-trihydroxybenzene by toluene 4-monooxygenase of Pseudomonas mendocina KR1 and toluene 3-monooxygenase of Ralstonia pickettii PKO1. Appl Environ Microbiol 2004, 70:3814-3820.

3. Robinson GK, Stephens GM, Dalton H, Geary PJ: The production of catechols from benzene and toluene by Pseudomonas putida in glucose fed-batch culture. Biocatal Biotransform 1992, 6:81-100.

4. Gurujeyalakshmi G, Oriel P: Isolation of phenol-degrading Bacillus stearothermophilus and partial characterization of the phenol hydroxylase. Appl Environ Microbiol 1989, 55:500-502.

5. Natarajan MR, Oriel P: Production of catechol by a Bacillus stearothermophilus transpositional mutant. Biotechnol Prog 1992, 8:78-80.

6. Draths KM, Frost JW: Conversion of D-glucose into catechol: the not-so-common pathway of aromatic biosynthesis. J Am Chem Soc 1991, 113:9361-9363.

7. Li W, Xie D, Frost JW: Benzene-free synthesis of catechol: interfacing microbial and chemical catalysis. J Am Chem Soc 2005, 127:2874-2882.

8. Balderas-Hernández VE, Sabido-Ramos A, Silva P, Cabrera-Valladares N, Hernández-Chávez G, Báez-Viveros JL, Martínez A, Bolívar F, Gosset G: Metabolic engineering for improving anthranilate synthesis from glucose in Escherichia coli. Microb Cell Fact 2009, 8:19.

9. Harwood CS, Parales RE: The beta-ketoadipate pathway and the biology of self-identity. Annu Rev Microbiol 1996, 50:553-590.

10. Costaglioli P, Barthe C, Claverol S, Brözel VS, Perrot M, Crouzet M, Bonneu M, Garbay B, Vilain S: Evidence for the involvement of the anthranilate degradation pathway in Pseudomonas aeruginosa biofilm formation. Microbiologyopen 2012, 3:326-339.

11. Bundy BM, Campbell AL, Neidle EL: Similarities between the antABC-encoded anthranilate dioxygenase and the ben $A B C$-encoded benzoate dioxygenase of Acinetobacter sp. strain ADP1. J Bacterio/ 1998, 180:4466-4474.

12. Kim D, Yoo M, Kim E, Hong SG: Anthranilate degradation by a cold-adapted Pseudomonas sp. J Basic Microbiol 2013, 53:1-9.

13. Yanofsky C, Horn V, Bonner M, Stasiowski S: Polarity and enzyme functions in mutants of the first three genes of the tryptophan operon of Escherichia coli. Genetics 1971, 69:409-433.

14. Báez JL, Bolívar F, Gosset G: Determination of 3-deoxy-Darabino- heptulosonate 7-phosphate productivity and yield from glucose in Escherichia coli devoid of the glucose phosphotransferase transport system. Biotechnol Bioeng 2001, 73:530-535.

15. Stover CK, Pham XQ, Erwin AL, Mizoguchi SD, Warrener P, Hickey MJ, Brinkman FSL, Hufnagle WO, Kowalik DJ, Lagrou M, Garber RL, Goltry L, Tolentino E, Westbrock-Wadman S, Yuan Y, Brody LL, Coulter SN, Folger KR, Kas A, Larbig K, Lim R, Smith K, Spencer D, Wong GKS, Wu Z, Paulsen IT, Reizer J, Saier MH, Hancock REW, Lory S, Olson MV: Complete genome sequence of Pseudomonas aeruginosa PAO1, an opportunistic pathogen. Nature 2000, 406:959-964.

16. Hernández-Montalvo V, Martínez A, Hernández-Chavez G, Bolivar F, Valle F, Gosset G: Expression of galP and glk in a Escherichia coli PTS mutant restores glucose transport and increases glycolytic flux to fermentation products. Biotechnol Bioeng 2003, 83:687-694.

17. Von Kamp A, Schuster S: Metatool 5.0: fast and flexible elementary modes analysis. Bioinformatics 2006, 22:1930-1931.

18. Panina EM, Vitreschak AG, Mironov AA, Gelfand MS: Regulation of aromatic amino acid biosynthesis in gamma-proteobacteria. $J$ Mol Microbiol Biotechnol 2001, 3:529-543. 
19. Bird PI, Pak SC, Worrall DM, Stephen P, Bottomley SP: Production of recombinant serpins in Escherichia coli. Methods 2004, 32:169-176.

20. Draths KM, Frost JW: Environmentally compatible synthesis of catechol from D-glucose. J Am Chem Soc 1995, 117:2395-2400.

21. Patnaik R, Spitzer RG, Liao JC: Pathway engineering for production of aromatics in Escherichia coli: confirmation of stoichiometric analysis by independent modulation of AroG, TktA, and Pps activities. Biotechnol Bioeng 1995, 46:361-370

22. Flores N, Xiao J, Berry A, Bolivar F, Valle F: Pathway engineering for the production of aromatic compounds in Escherichia coli. Nat Biotechnol 1996, 14:620-623.

23. Caligiuri MG, Bauerle R: Identification of amino acid residues involved in feedback regulation of the anthranilate synthase complex from Salmonella typhimurium. Evidence for an amino-terminal regulatory site. J Biol Chem 1991, 266:8328-8335.

24. Sørensen HP, Mortensen KK: Soluble expression of recombinant proteins in the cytoplasm of Escherichia coli. Microb Cell Fact 2005, 4:1.

25. Singh SP, Purohit MK, Aoyagi C, Kitaoka M, Hayashi K: Effect of growth temperature, induction, and molecular chaperones on the solubilization of over-expressed cellobiose phosphorylase from Cellvibrio gilvus under in vivo conditions. Biotechnol Bioprocess Eng 2010, 15:273-276.

26. Schweigert N, Zehnder AJ, Eggen RI: Chemical properties of catechols and their molecular modes of toxic action in cells, from microorganisms to mammals. Environ Microbiol 2001, 3:81-91.

27. Sun $X$, Lin $Y$, Huang $Q$, Yuan $Q$, Yan Y: A novel muconic acid biosynthesis approach by shunting tryptophan biosynthesis via anthranilate. Appl Environ Microbiol 2013, 79:4024-4030.

doi:10.1186/s12934-014-0136-x

Cite this article as: Balderas-Hernández et al: Catechol biosynthesis from glucose in Escherichia coli anthranilate-overproducer strains by heterologous expression of anthranilate 1,2-dioxygenase from Pseudomonas aeruginosa PAO1. Microbial Cell Factories 2014 13:136.

\section{Submit your next manuscript to BioMed Central and take full advantage of:}

- Convenient online submission

- Thorough peer review

- No space constraints or color figure charges

- Immediate publication on acceptance

- Inclusion in PubMed, CAS, Scopus and Google Scholar

- Research which is freely available for redistribution 